\title{
BMJ Open Cancer treatment decision-making processes for older patients with complex needs: a qualitative study
}

\author{
Jackie Bridges, Jane Hughes, Naomi Farrington, Alison Richardson
}

To cite: Bridges J, Hughes J, Farrington $\mathrm{N}$, et al. Cancer treatment decision-making processes for older patients with complex needs: a qualitative study. BMJ Open 2015;5:e009674.

doi:10.1136/bmjopen-2015009674

- Prepublication history for this paper is available online. To view these files please visit the journal online (http://dx.doi.org/10.1136/ bmjopen-2015-009674).

Received 10 August 2015 Revised 30 September 2015 Accepted 23 October 2015

CrossMark

Faculty of Health Sciences, University of Southampton, Southampton, UK

Correspondence to Professor Jackie Bridges; jackie.bridges@soton.ac.uk

\section{ABSTRACT}

Objectives: Although older people can experience complex health and social care needs alongside a primary cancer diagnosis, little is understood about how cancer treatment decisions are made for this population. This study aimed to investigate how cancer treatment decisions are formulated for older people with complex health and social care needs and the factors that shape these processes.

Design: Qualitative study involving semistructured interviews and non-participant observations. Framework approach used for data analysis.

Setting: Breast and colorectal cancer services in five English NHS hospital trusts.

Participants: Interviews: purposive sample of 22 clinicians directly involved in a face-to-face clinical role with patients regarding cancer treatment and care, maximising variation across clinical roles, tumour types and trusts. Observations: purposive sample of five cancer multidisciplinary meetings, maximising variation across location, team size and tumour type.

Results: The initial stages of cancer treatment decision-making are team-based, medically dominated and focused on the cancer. For patients with complex health and social care needs that extend beyond cancer pathology, later and less visible stages in the decisionmaking process are more haphazard and may result in less effective and workable treatment plans, as individual clinicians struggle to devise and deliver these plans without breaching time-based targets.

Conclusions: Service targets that focus resources solely on the presenting disease can disadvantage older patients with complex health and social care needs that extend beyond this primary diagnosis. Care should be taken to ensure time-based targets do not disincentivise thorough and timely assessment that can lead to the formulation of treatment plans tailored to individual needs and circumstances.

More than a third of cancers are diagnosed in people aged 75 years and above, but low UK survival rates for older people have in recent years been identified as a matter of significant concern, with evidence suggesting a range of causative factors. ${ }^{1-3}$ Recent attention has been directed at the influence of chronological age on treatment options

\section{Strengths and limitations of this study}

- This is the first report on the characteristics of cancer treatment decision-making for older patients with cancer, and the contextual factors that influence these processes.

- The in-depth qualitative study across five English NHS Trusts draws attention to and deepens our understanding of the complex processes that can be involved in making a treatment decision, and examines the appropriateness of a linear, medically focused pathway underpinned by strong time-based incentives.

- The research on which this paper is based was originally designed to evaluate a specific intervention and this may have influenced how respondents chose to present themselves and their service.

- The use of non-participation observation alongside semistructured interviews enabled us to validate clinicians' accounts of multidisciplinary meetings against what was observed.

- This study did not explore patient perceptions of the treatment decision-making processes.

presented to older patients with cancer by clinicians, with studies suggesting increased age can limit the options presented even though age alone is widely recognised to be an inadequate indicator of an individual's capacity for cancer treatment tolerance. ${ }^{2}{ }^{4-6}$ Clinician anxieties about treatment toxicity, side effects and burden may be an important constraining influence on the intensity of cancer treatment offered to older people, ${ }^{78}$ but other more subtle factors may also influence treatment decisions. This paper presents research findings that illuminate cancer treatment decision-making processes for older people with complex needs.

Studies of cancer MDT (multidisciplinary team) meetings to date (not focusing on older people) indicate that clinicians focus on one aspect of the decision-making process (the cancer pathology) in order to make the work easier to organise and 
control, thereby standardising by selection. ${ }^{9}$ Cohen draws attention to the difficulties involved in attempts to organise and control 'body work' such as healthcare, that is paid work that involves human bodies. ${ }^{9}$ Certain processes can serve the function of producing more predictable and malleable work, thus creating opportunities to organise and rationalise. One such process is standardisation by selection and examples include selecting by body type or focusing on a single body part. Research on cancer MDTs to date has highlighted markedly less consideration in MDT discussions of other factors such as comorbidities or patient choice, ${ }^{10-12}$ and this focus indicates that standardisation by selection could be a key feature of cancer MDT meetings.

Owing to increased propensity for comorbidities and associated frailty, older people are more likely to have health and social care concerns that extend beyond their cancer pathology, these concerns sometimes affecting treatment tolerance and requiring a modified treatment plan and relevant supportive care. The more complex the needs, the higher the risk of poor outcomes that can stem from processes and decisions that only focus on the cancer pathology and that do not take account of factors such as frailty, comorbidity or social situation. ${ }^{13}{ }^{14}$ However, we understand little about how clinicians and patients arrive at cancer treatment decisions in practice, and the system features that shape these processes. MDT treatment decisions may be reformulated following the MDT, ${ }^{15-17}$ underlining the importance of exploring processes outside of formal MDT meetings.

Drawing on qualitative data from a wider evaluation project, this paper presents an insight into how cancer treatment decisions are made for older people with complex needs, and the system features that shape these decision-making processes. While health systems internationally vary in the extent to which specialist services for older people with cancer have been developed, the relevance of this UK case study lies in the analysis of the impact of system features such as external performance targets on the effectiveness of professional work in relation to complex patient need.

\section{METHODS}

The research reported here was conducted as part of a wider mixed methods feasibility study of comprehensive geriatric assessment (CGA) ${ }^{18}$ for older patients with cancer across a group of five English NHS hospital trusts. ${ }^{2}$ The qualitative approach described below, using interviews and direct observation, was used to gather data in the larger study on cancer treatment decisionmaking processes for older people with complex needs, theorised as a factor that could influence implementation of CGA. A qualitative approach was selected to enable an in-depth exploration of these social phenomena in their natural setting. ${ }^{19}$

\section{Setting and context}

All five participating hospital trusts provided breast and colorectal cancer services in a range of settings from local district general hospitals to regional specialist centres. Multidisciplinary meetings, organised by tumour type, took place in each trust, with remote access to oncologists at specialist centres during team meetings held in hospitals geographically distant from the specialist centre. One exception to this was a joint meeting held between staff from breast cancer services across three hospital trusts.

UK health policy mandates the use of a standardised cancer treatment pathway aimed at improving survival rates through reducing variability in treatment decisionmaking. ${ }^{20}$ The pathway includes time-based targets for each stage (figure 1) and the management of all cases by a designated MDT of specialists in the relevant cancer type.

\section{Sample}

Fifty-seven eligible healthcare professionals were identified and invited to take part. The purposive sampling strategy aimed to include people directly involved in a face-to-face clinical role with patients regarding their cancer treatment and care, maximising variation across clinical roles, tumour types and trusts. Twenty-two clinicians agreed to take part (table 1). All participants interviewed at the outset of the project were invited to a second interview at close of project to explore if responses changed over time. Five nurse specialists, one surgeon and one oncologist were interviewed twice, on average 11 months after the first interview.

\section{Data collection}

Twenty telephone interviews (average length $45 \mathrm{~min}$ ) and four group interviews $(n=2-4$ per interview, average length $50 \mathrm{~min}$ ) were conducted by $\mathrm{JB}$ and $\mathrm{JH}$. Open-ended questions were used to stimulate respondents to discuss their experiences of, and views on, current service provision for older people with cancer, on decision-making about treatment for older people with cancer, and the CGA project. All interviews were audio recorded; and recordings were subsequently transcribed verbatim.

Five complete MDT meetings were also observed (over $8 \mathrm{~h}$ in total) each at a different hospital site. Two meetings focused on breast cancer and three on colorectal cancer. Non-participant observation was carried out by a single researcher (JB) using an abridged version of the MDT-OARS (Observation Assessment Rating Scale), a structured observational tool developed for measuring the quality of MDT working. ${ }^{21}$ Data were gathered in-vivo on the team (attendance, chairing and teamworking); infrastructure for meetings (venue and equipment); meeting organisation and logistics; and patient-centred clinical decision-making (patient centredness, information used to inform decisions, clarity of recommendations). Notes taken in the 
Figure 1 Diagnostic care pathway for cancer.

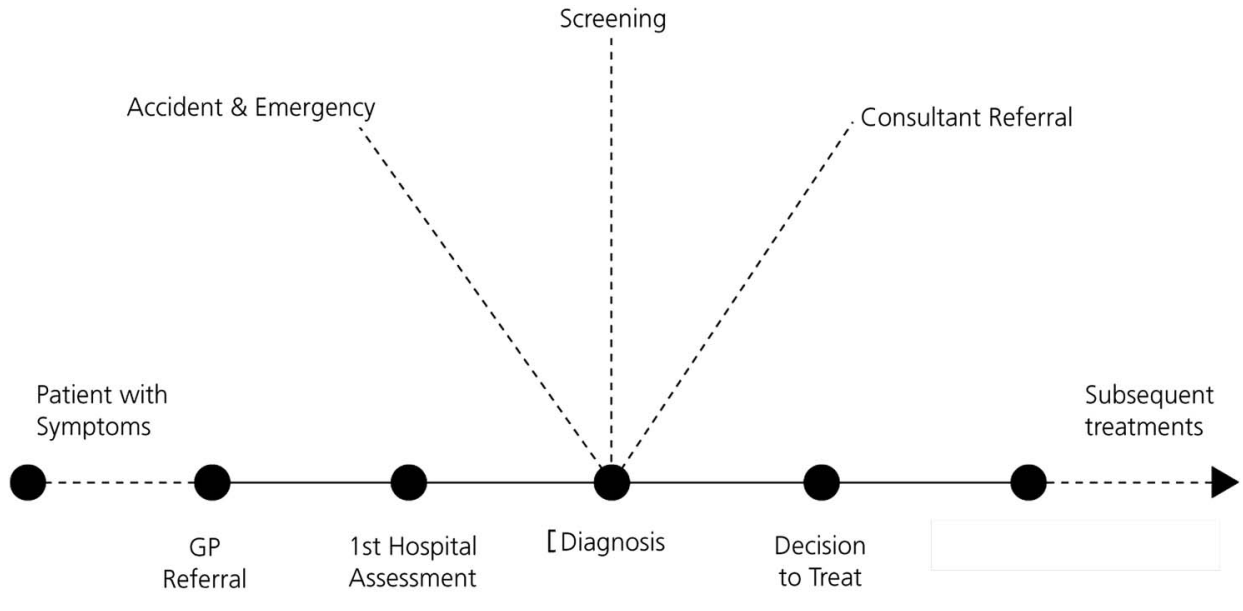

meeting were supplemented by comprehensive qualitative field notes audio recorded immediately following the meeting and later transcribed.

Data were gathered between July 2011 and September 2012 by researchers who were not otherwise connected with the individuals in the study. JB is a health services researcher and registered nurse specialising in social research on professional work, organisational change and older people's care. $\mathrm{JH}$ is a social scientist with a special interest in health services research.

\section{Data analysis}

The framework approach to analysis was used. ${ }^{22}{ }^{23} \mathrm{An}$ initial framework was agreed between the two coders for indexing the data, including a priori categories determined by the study focus on making treatment decisions, and themes that were derived inductively from the data. Using the constant comparative method, the data were indexed and charted for each key theme, enabling comparison between participants, and where appropriate triangulation of data from interviews and direct observation. Summary charts, giving brief accounts of respondents' views, noting similarities and contrasts with illustrative quotes, formed the basis for selective elaboration and further refinement of relevant themes.

\section{Ethical approval}

Ethical approval for the study was obtained from NRES Committee London-City Road and Hampstead, REC reference 11/HO721/10. All interviewees provided

\begin{tabular}{llllc} 
Table 1 & Sample characteristics & & \\
\hline & $\begin{array}{l}\text { Consultant } \\
\text { surgeons }\end{array}$ & $\begin{array}{l}\text { Consultant } \\
\text { oncologists }\end{array}$ & $\begin{array}{l}\text { Nurse } \\
\text { specialists }\end{array}$ & Total \\
\hline Trust 1 & 1 & 0 & 2 & 3 \\
Trust 2 & 0 & 4 & 2 & 6 \\
Trust 3 & 1 & 2 & 4 & 7 \\
Trust 4 & 2 & 0 & 2 & 4 \\
Trust 5 & 1 & 0 & 1 & 2 \\
Total & 5 & 6 & 11 & 22 \\
\hline
\end{tabular}

written informed consent. Observations took place with written permission of the meeting chair and advance provision of information to team members.

\section{RESULTS}

Respondents described decision-making about treatment for all patients with cancer as a process, beginning at the MDT meeting and continuing throughout the cancer treatment pathway:

So it starts right from the beginning about what sort of treatment they're going to have. And then there continue to be a number of choice points along the way. (Oncologist D03) ${ }^{\mathrm{i}}$

\section{A focus on the cancer pathology in MDT meetings}

Typically present at the MDT meetings observed were surgical teams, oncologists and nurses directly involved with patient cancer care and treatment, together with radiologists and pathologists who provided the investigation results required to make a diagnosis and inform treatment plans. Respondents perceived the MDT meeting as providing a structured and rigorous approach to reviewing investigation results and reaching a diagnosis, enabling the formulation of a treatment plan that represented the optimum treatment for the pathology. MDT meetings were described as focusing on cancer pathology rather than the patient:

So it's quite a lot to get through, 'cos we have to look at the pathology, imaging. It has to be very medical and almost devoid of the patient. (Surgeon D08)

This perspective corresponded with our observation of MDT meetings. For patients with a new diagnosis of cancer, radiology and histopathology findings were presented to determine the diagnosis and develop treatment recommendations. Information about comorbidity was sometimes part of the observed discussions but not

${ }^{\mathrm{i}} \mathrm{D}$ denotes Doctor and the number denotes order of enrolment into study. 
routinely included. Interviewees said that it was generally assumed by meeting participants that if a patient had significant problems these would be recorded on the meeting notes or mentioned, although sometimes this information was not available.

Sometimes the doctor who's seen the patient isn't necessarily at the meeting or the nurse may not be there, so you may be discussing the patient with the notes rather than somebody who's familiar with the patient. So I think that makes it difficult ... to make an all-round balanced decision. (Oncologist D10)

Yes, because some decisions we make at the [MDT meeting] we would say "We are presuming this patient is very fit" and then we say "This patient will have chemotherapy/radiotherapy and whatever". And then they come to the clinic and you find that their blood sugar is out of control, or they've had a recent stroke, or whatever. And then that changes everything (Oncologist D06)

In our MDT, very few social issues are being discussed, if I'm truthful. (Oncologist D07)

It was rare in meetings observed for anyone to introduce more complex patient-centred information, such as comorbidities, psychosocial and supportive care needs or patient preferences. Medical and nursing staff talked about the importance of nurses' input to bring these wider issues to the attention of the meeting:

The breast care nurses do all attend the MDT meeting, and they recognise their role as patient advocates, and they are listened to and respected. (Surgeon D03)

You know after diagnosis you can get a sense sometimes of people, of what their initial thoughts might be on how they may want to have treatment or not have treatment, or be amenable to this but not to that, and you can kind of enter that into part of the MDT meeting discussion. (Nurse $\mathrm{N} 4^{\mathrm{ii}}$ )

However, a consistent nursing contribution was not observed across the meetings. In one meeting, a nurse specialist presented patient cases and took the lead in coordinating a plan of action for them. However, in other meetings observed, nurses rarely participated in discussions.

\section{National targets dictate pace and style of meeting}

Respondents cited the powerful impact of the national cancer targets that dictated the time periods between referral, diagnosis and treatment, and required every patient with suspected cancer to have their case discussed at an MDT meeting. Respondents said the large number of patients scheduled for discussion constrained

\footnotetext{
${ }^{\mathrm{ii}} \mathrm{N}$ denotes Nurse.
}

the length of debate. Presentations had to be slick and decisions swift.

We have a very, very big multidisciplinary meeting, which is under enormous time pressure. So it often has to be very business-like, and this is this, this is that, this is the treatment. Next! Just purely because of the sheer numbers, and the softer stuff has to be left for later. (Surgeon D08)

At each observed MDT meeting participants adopted a pace and style that enabled them to get through all the patients on the agenda within the time allocated for the meeting, with average presentation and discussion time per patient ranging from $2.4 \mathrm{~min}$ in one breast cancer MDT meeting to $5.6 \mathrm{~min}$ in a colorectal meeting. A small number of patients were discussed at some length and these extended discussions were commonly prompted by clinical complexity related to the cancer.

\section{MDT recommendations are first stage in treatment decision-making}

Most interviewees said they would have liked sufficient information and time to allow more patient-centred discussion at the MDT meeting, but they did not necessarily consider the meeting's narrow focus on cancer pathology as a major deficiency or problem. A treatment plan outlined at an MDT meeting was seen by respondents as provisional or contingent because it was based on an incomplete clinical picture of the patient, and MDT decisions were typically referred to in interviews as recommendations, to be taken forward by clinicians to the next stage of the decision-making process.

I think we probably will say "Yes, this patient might be suitable for treatment $\mathrm{A}$, but we need to see them in clinic and set our eyes on them and assess those sort of other factors with the patient in front of us." (Oncologist D10)

Subsequent decision-making included (re)assessing the patient's comorbidities, general fitness and ability to cope with treatment; and it was at this point that patients became involved in decision-making.

The findings above illustrate the first stage of cancer treatment decision-making and the characteristics of the context in which these decisions are taken. These preliminary decisions, seen as contingent on later patient consultation and information, are made primarily by medical staff on the basis of the cancer pathology together with other relevant information if it is available. The requirement that everyone with a potential cancer diagnosis is discussed at an MDT meeting means that time is limited for discussion of individual cases. The findings that follow illustrate how, outside MDT meetings, further information is gathered and used to inform discussions between clinicians and patients about treatment decisions, and the factors that influence these processes. 
Lack of time constrains meaningful patient involvement in decision-making

Respondents expressed a desire to involve people with cancer in making decisions about their treatment, but felt older patients needed more time for assessment and discussion of treatment options. Busy clinic schedules could disadvantage older patients, as well as testing doctors' interpersonal skills:

I think the idea of seeing the elderly person as a person, not just "the elderly", is important. You could try and understand where they're coming from; see it from their viewpoint... But we all too easily end up just seeing some old, grey, wrinkly person in the clinic, who takes too long to get dressed and undressed; who doesn't quite arrive at the answer as quick as we want them to. And then they don't quite fit in with treatment plans and it takes longer to sort them out, and it all becomes a bit of a nuisance. (Surgeon D08)

Nurses thought that the way some consultants presented treatment options could influence older patients' decisions and they tried to compensate by giving patients additional information and opportunities for discussion.

I think for the older generation, a lot of them, doctor is God I think, you know...I think the patient might be a bit more reluctant to ask questions, and then it will all come afterwards, and they'll come out with all these things once the doctor has gone. (Nurse N5)

Nurses sought to form trusting relationships with older patients to enable continuity of care and facilitate access to support during treatment. However, older people were characterised as resistant to professional attempts to establish a relationship; and in some narratives they were positioned as private, even uncooperative.

People of that generation can be very private sometimes. They are reluctant to share information. (Nurse N8)

Some people are remarkably resistant to letting us in... they are a minority, and so yes, making that assessment of them, it often develops over a period of time in that they decide whether they get to trust us, and then give us information, or whether they have such pre-formed ideas about what doctors do and so on that they are either not going to. (Oncologist D03)

Clinicians consistently cited the influence of time pressures in the cancer treatment pathway that determined when treatment should begin, driving the decisionmaking process and determining their work schedules. Most respondents wanted to enable patients to make choices about treatment but could not always allow them to do so at their own pace because of national targets that dictated the maximum length of time between diagnosis and the start of treatment.
One possible problem is because of the need to treat people quickly. There's not a lot of time often for very big decisions to be made; you've literally just got a couple of weeks, depending on what pathway a patient is on, you haven't got a lot of time really. You've got to squeeze the test in, get all the results, you have a discussion with the patient, and get them treated, or else, you are told they are going to breach [the national time-based target for treatment], and obviously get into trouble for breaching. So I think with the more complex decisions, if someone's going to perhaps have a permanent colostomy, it is difficult. And most people cope very, very well, but it's of course a big change to their life... but you are under the pressure of time to make these decisions. (Nurse N9)

Professionals having time to build relationships with patients were identified as critical to enabling optimal involvement in decisions and in the best treatment decision for that individual being formulated, but a lack of time dictated by the national targets meant these decisions often felt pressured. This constrained the meaningful involvement of patients in decision-making about their treatment.

\section{Pertinent patient information not always available in time for treatment decisions}

One important feature identified by interviewees as shaping treatment decision-making was the quality, availability and timeliness of information about the patient. Respondents talked about the importance of assessing a patient's fitness for treatment and agreed that assessment of older patients should include a wider range of issues. However, there was substantial variation in how fitness was defined and in the kind of additional patient information individual clinicians gathered and used to inform decisions about older people's cancer treatment and care.

There's no formal assessment tool in use, but I think we do-I think as nurses you do an awful lot of activities of daily living, assess the person, and what's going on with them and their lives at that time. And then kind of highlighting things for you, and just through your experience you try and reach the point where you hold that information for later on. (Nurse N4)

Respondents described relying on clinical experience to identify patients who were frail, needed further assessment, or whose suitability for particular treatment options was in doubt.

And a lot of it, we just think we know, ...rather than having a 20 page tool to assess the patient's functional, cognitive, aspect of their life, we kind of go on a hunch or... our sort of clinical expertise about how a patient is functioning and coping. (Oncologist D09)

Assessment of cognitive impairment was singled out as being particularly unsatisfactory, with clinicians not feeling confident in their abilities to detect it. 
When patients come to clinics, because of lack of time we often don't pick up [cognitive impairment]. [Older patients] answer the questions correctly, and we think they're fine, they can go ahead with whatever treatment. And then we find during the treatment they are forgetting things, they are not going to their appointments, or they went to the wrong appointment... These are people who are not known to have dementia, but there are a lot of people out there who have memory problems... So we all think that we are treating patients who are fit....and they are not as fit as we think. (Oncologist D06)

All the oncologists interviewed wanted to find ways of assessing older patients that overcame inherently subjective judgments of 'fitness', especially for chemotherapy. Most interviewees thought a more consistent approach to assessment would benefit patients.

I see elderly patients who we think are fit enough for treatment...then they suffer from considerable side effects and we have to curtail treatment or reduce the dose, and this ultimately will impact on outcomes. (Oncologist D10)

The imperative to start treatment within a certain time period constrained any flexibility that may have been needed to assess people thoroughly, and any further multidisciplinary input needed to their treatment plan and its execution.

You almost need another MDT meeting to bring that patient back to discuss those assessments that you have done. Because the chances are you are going to do those assessments at diagnosis, by which time they've usually already been to the MDT meeting. They may go back once more, but they are not always likely to, you know. So there isn't really going to be another opportunity when the whole team is going to be able to use the benefit of those assessments. (Nurse N7)

If my care of the elderly colleagues find that Patient $\mathrm{X}$ needs a lot more social input, and that would increase their chances of taking up radiotherapy or chemotherapy, the problem is that there's a clock ticking on this, from an oncology point of view, which I have to start treatment within $\mathrm{X}$ number of days or we breach. (Oncologist, D04)

Respondents in both sets of interviews reported that a lack of timely information meant additional work for clinicians, often at short notice, to fill gaps and make connections to ensure that a patient's treatment could proceed as planned. Nurses frequently undertook this type of compensatory work to enable patients to fit into processes. They described having to respond swiftly using personal contacts and unofficial channels, to make sure older patients were not disadvantaged and targets were met.

So it's got to be a fairly rapid scramble in order to get services in if you are going to need them. (Nurse N7)
We cheat...we'll give them a provisional date for surgery, and then it gives us like 2 weeks or so to run around and get things done. (Nurse N2)

The importance of knowing about patient's wider health and social care needs was acknowledged by all the clinicians interviewed, but our findings illustrate the difficulties they experienced in ensuring this information was available in time to inform the cancer treatment decision. Constraints identified included absence of consensus about what information was required and who should gather it, and poorly developed clinical skills in identifying issues such as cognitive impairment. Once information was available, clinicians needed to engage in rushed compensatory work using informal networks to address the issues identified within the timeframe determined by the treatment targets.

\section{DISCUSSION}

Our findings show that the cancer treatment-decisionmaking processes that immediately follow diagnosis take place in a team and can be characterised as medically dominated and narrowly focused on cancer pathology. The treatment recommendations formulated are then presented to the patient by an individual clinician, the patient's views being considered along with any patient details that subsequently emerge including the results of clinicians' and others' assessments. At this and later points, should further pertinent information become available, the original treatment recommendation can be revised by the individual clinician outside of the MDT meeting. In some, but not all, cases the patient's case is reviewed at a later MDT meeting in the light of further information that becomes available. These findings, particularly those that illustrate cancer team decision-making are consistent with findings from other studies. $^{10-12}$

However, our findings also unveil a rich picture of behind-the-scenes decision-making and associated work when patient need is complex. These findings may help explain why older people with cancer in the UK have poorer outcomes. ${ }^{1}{ }^{2}$ Relevant information about these wider needs may not emerge in time for MDT discussion, or may emerge later and be hostage to individual clinicians who may not possess skills to work these issues into the treatment plan or the capacity to make the necessary onward referrals. It is also possible that relevant information may not become available until the treatment plan is implemented, if at all. The more complex the patient needs, the higher the chance that the resulting treatment plan will be ineffective or unworkable. The additional work by doctors and nurses outside of meetings to compensate for this lack of fit between these processes and patients with complex needs may go some way towards ameliorating this situation. However, individual clinicians found the nature of the MDT meetings and the pressures of the 'ticking 
clock' constrained their capacity to respond appropriately to complex patient needs, constraints that intensified as patient needs became more complex and diverse.

Current decision-making processes are strongly shaped by national cancer targets, from the style and purpose of MDT meetings to the speed at which individual patients are expected to progress to treatment. These time targets reinforce a narrow view of complex clinical work by focusing on one aspect (the cancer pathology) and obscuring others. This standardisation by selection allows MDT meetings to proceed in a predictable fashion and expedites decision-making, enabling the work to be accomplished within the time allotted. ${ }^{9}$ It also determines who needs to be in the room, and limits discussions with potential to extend beyond the cancer.

Other studies have highlighted how targets specified by national government can focus attention and resources on particular priorities, but that this focus can then detract from other aspects of service quality and safety. ${ }^{24-27}$ Our findings confirm this analysis and illustrate the impact on frontline patient care. They show how working practices, in less well illuminated reaches of the cancer pathway, are pivotal to the process of making treatment decisions for patients with cancer with complex health and social care needs, and yet these practices and the resulting decisions are not scrutinised or made visible, unless the individual clinician decides to consult MDT colleagues. MDTs are positioned in policy as the locus of decision-making about treatment and care, but we found that for more complex patients, decisions could be made later by individual clinicians who struggled to involve older people in a meaningful way and who may not have all the relevant information to hand. A range of factors shaped treatment decisions, including whether relevant information was sought, shared and used; the capacity and skill of individual clinicians; the effectiveness of links with specialists such as geriatric physicians; and whether there was sufficient time to develop and put in place the optimal treatment plan.

The strengths of this study lie in the rich interview and observational data generated over a 15-month period from services for two common tumour types in a large and diverse group of hospitals. The research on which this paper is based was originally designed to evaluate a specific intervention and this may have influenced how respondents chose to present themselves and their service. However, their narratives articulate constraints on the formulation of effective treatment decisions for older patients with complex needs. We have illustrated how time-based measures of success can reduce capacity and incentive to assess patients' wider needs, yet a timely and thorough assessment is the cornerstone of a tailored and workable treatment plan. As cancer strategies are reformulated to reflect increasingly complex patient populations, attention should be paid to defining which patients would benefit from more comprehensive assessment and what factors should be assessed, in addition to clarifying who is best placed to conduct the assessments, and at what point geriatric and other specialist services need linking in. Reflection on the findings from assessments with the patient and relevant specialists should be adequately resourced, as should the provision of practical and social support services to enable patients to take up their recommended treatment plan.

Attention should now shift towards exploring how decision-making processes could be modified and structural support provided to ensure that patients with cancer with complex needs receive adequate and timely assessment and access to clinical experts with the capacity to support them to arrive at the best treatment decision.

Twitter Follow Jackie Bridges at @JackieLearning

Acknowledgements The authors would like to thank all the participants who participated in this study and all the staff at Macmillan Cancer Support and the Cancer Network who contributed to its execution and completion through facilitating access for the research team and providing critical commentary on emerging findings. The authors also thank Dr Michael Glynn and journal reviewers for their comments on earlier versions of the manuscript.

Contributors JB and AR designed the study. JB and JH collected data. JB, JH and $A R$ analysed the data. JB and JH drafted the work. JB, AR, NF and JH revised the work critically for important intellectual content. JB, AR, NF and $\mathrm{JH}$ approved the final version for publication.

Funding This work was supported by Department of Health (England) and Macmillan Cancer Support, but the findings presented are independent of influence from these organisations.

Competing interests None declared.

Ethics approval NRES Committee London-City Road \& Hampstead.

Provenance and peer review Not commissioned; externally peer reviewed.

Data sharing statement No additional data are available.

Open Access This is an Open Access article distributed in accordance with the Creative Commons Attribution Non Commercial (CC BY-NC 4.0) license, which permits others to distribute, remix, adapt, build upon this work noncommercially, and license their derivative works on different terms, provided the original work is properly cited and the use is non-commercial. See: http:// creativecommons.org/licenses/by-nc/4.0/

\section{REFERENCES}

1. Office for National Statistics. Statistical Bulletin, Cancer Survival in England: Adults Diagnosed 2008 to 2012, followed up to 2013. ONS, 2014. http://www.ons.gov.uk/ons/dcp171778_382524.pdf (accessed 7 Aug 2015).

2. Department of Health. Cancer services coming of age: learning from the improving cancer treatment assessment and support for older people project. Macmillan Cancer Support, 2012. http://www. macmillan.org.uk/Documents/AboutUs/Health_professionals/ OlderPeoplesProject/CancerServicesComingofAge.pdf (accessed 15 Sep 2015).

3. Cancer Research UK. Cancer incidence by age- UK statistics. http:// www.cancerresearchuk.org/health-professional/cancer-statistics/ incidence/age\#ref-0 (accessed 29 Sep 2015).

4. Ring $A$. The influences of age and co-morbidities on treatment decisions for patients with HER2-positive early breast cancer. Crit Rev Oncol Hematol 2010;76:127-32. 
5. Walko CM, McLeod HL. Personalizing medicine in geriatric oncology. J Clin Oncol 2014;32:2581-6.

6. Mandelblatt JS, Jacobsen PB, Ahles T. Cognitive effects of cancer systemic therapy: implications for the care of older patients and survivors. J Clin Oncol 2014:32:2617-26.

7. National Cancer Equality Initiative/Pharmaceutical Oncology Initiative. The impact of patient age on clinical decision-making in oncology. London: Department of Health, 2012.

8. Kalsi T, Babic-Illman G, Fields $P$, et al. The impact of low-grade toxicity in older people with cancer undergoing chemotherapy. Br J Cancer 2014;111:2224-8.

9. Cohen RL. Time, space and touch at work: body work and labour process (re)organisation. Sociol Health IIIn 2011;33 189-205.

10. Kidger J, Murdoch J, Donovan J, et al. Clinical decision-making in a multidisciplinary gynaecological cancer team: a qualitative study. BJOG 2009;116:511-17.

11. Lamb BW, Brown KF, Nagpal K, et al. Quality of care management decisions by multidisciplinary cancer teams: a systematic review. Ann Surg Oncol 2011;18:2116-25.

12. Jalil R, Ahmed M, Green JS, et al. Factors that can make an impact on decision-making and decision implementation in cancer multidisciplinary teams: an interview study of the provider perspective. Int J Surg 2013;11:389-94.

13. Handforth C, Clegg A, Young C, et al. The prevalence and outcomes of frailty in older cancer patients: a systematic review. Ann Oncol 2015;26:1091-101.

14. Chen RC, Royce TJ, Extermann M, et al. Impact of age and comorbidity on treatment and outcomes in elderly cancer patients. Semin Radiat Oncol 2012;22:265-71.

15. Blazeby J, Wilson L, Metcalfe C, et al. Analysis of clinical decision-making in multi-disciplinary cancer teams. Ann Oncol 2006;17:457-60.
16. English R, Metcalfe C, Day J, et al. A prospective analysis of implementation of multi-disciplinary team decisions in breast cancer. Breast J 2012;18:459-63.

17. Wood J, Metcalfe C, Paes A, et al. An evaluation of treatment decisions at a colorectal cancer multi-disciplinary team. Colorectal Dis 2008;10:769-72.

18. Wildiers $\mathrm{H}$, Heeren $\mathrm{P}$, Puts $\mathrm{M}$, et al. International society of geriatric oncology consensus on geriatric assessment in older patients with cancer. J Clin Oncol 2014;32:2595-603.

19. Pope C, Mays N. Qualitative research in health care. John Wiley \& Sons, 2008.

20. Department of Health. The NHS cancer plan: a plan for investment, a plan for reform. London: Department of Health, 2000.

21. Williams CL, Hyer K, Kelly A, et al. Development of nurse competencies to improve dementia care. Geriatr Nurs 2005;26:98-105.

22. Ritchie J, Spencer L. Qualitative data analysis for applied policy research. In: Bryman A, Burgess RG, eds. Analysing qualitative data. London: Routledge, 1994:173-94.

23. Pope C, Ziebland S, Mays N. Qualitative research in health care: analysing qualitative data. BMJ 2000;320:114.

24. Bridges J, Fitzgerald L, Meyer J. New workforce roles in health care: exploring the longer-term journey of organisational innovations. $J$ Health Organ Manag 2007;21:381-92.

25. Fitzgerald L, Lilley $\mathrm{C}$, Ferlie $\mathrm{E}$, et al. Managing change and role enactment in the professionalized organization. London: NCCSDO, 2006.

26. Ross F, Christian S, Clayton J, et al. The professional experience of governance and incentives: meeting the needs of individuals with complex conditions in primary care. London: SDO, 2009.

27. Bridges $\mathrm{J}$, Meyer J. Exploring the effectiveness of action research as a tool for organisational change in health care. $J$ Res Nurs 2007;12:389-99. 